University of Nebraska - Lincoln

DigitalCommons@University of Nebraska - Lincoln

Ralph Skomski Publications

Research Papers in Physics and Astronomy

February 2008

\title{
Band-structure and correlation effects in the Co(111) planes of $\mathrm{CoO}$
}

\author{
Ralph Skomski \\ University of Nebraska-Lincoln, rskomski2@unl.edu \\ Xiaohui Wei \\ University of Nebraska-Lincoln \\ David J. Sellmyer \\ University of Nebraska-Lincoln, dsellmyer@unl.edu
}

Follow this and additional works at: https://digitalcommons.unl.edu/physicsskomski

Part of the Physics Commons

Skomski, Ralph; Wei, Xiaohui; and Sellmyer, David J., "Band-structure and correlation effects in the Co(111) planes of CoO" (2008). Ralph Skomski Publications. 49.

https://digitalcommons.unl.edu/physicsskomski/49

This Article is brought to you for free and open access by the Research Papers in Physics and Astronomy at DigitalCommons@University of Nebraska - Lincoln. It has been accepted for inclusion in Ralph Skomski Publications by an authorized administrator of DigitalCommons@University of Nebraska - Lincoln. 


\title{
Band-structure and correlation effects in the Co(111) planes of CoO
}

\author{
Ralph Skomski, ${ }^{a}$ Xiaohui Wei, and D. J. Sellmyer \\ Department of Physics and Astronomy and Nebraska Center for Materials and Nanoscience, \\ University of Nebraska, Lincoln, Nebraska 68588, USA
}

(Presented on 9 November 2007; received 11 September 2007; accepted 16 October 2007; published online 13 February 2008)

\begin{abstract}
The origin of the striking magnetic anisotropy of cobaltous oxide $(\mathrm{CoO})$ is investigated by model calculations. A key feature of the electronic structure of the antiferromagnetic compound is the accommodation of Co $3 d$ electrons in the (111) Co planes, with parallel and antiparallel intra- and interplane spin orientations and reminiscent of $\mathrm{Cu} 3 d$ electrons in the $\mathrm{CuO}_{2}$ planes in high-temperature superconductors. The strong correlations of the Co $3 d$ electrons lead to a picture intermediate between traditional crystal-field and itinerant-electron descriptions of the magnetic anisotropy. Using a simple configuration-interaction approach, we analyze the effect of $3 d^{8}-3 d^{6}$ bonds and their interplay with $3 d^{7}-3 d^{7}$ configurations. (C) 2008 American Institute of Physics.
\end{abstract}

[DOI: 10.1063/1.2830958]

Cobaltous oxide, $\mathrm{CoO}$, is an interesting antiferromagnetic (AFM) material, long investigated but comparatively little understood. Its relatively large magnetocrystalline anisotropy (of the order of $1 \mathrm{MJ} / \mathrm{m}^{3}$ ) stabilizes the exchange bias in $\mathrm{Co} / \mathrm{CoO}$ two-phase magnets, and its transport behavior has attracted much attention due to the Mott localization of the Co $3 d$ electrons. While not suitable for permanent magnets, the understanding of its anisotropy is important for other applications, such as magnetic recording and sensors.

The oxide crystallizes in the cubic rocksalt structure, and its AFM spin structure is realized by the (111) Co planes with ferromagnetic order in each plane but antiferromagnetic spin alignment between adjacent planes. ${ }^{1}$ Figure 1 shows the corresponding spin structure. Furthermore, there is a slight tetragonal distortion along the $c$ axis, which is also the uniaxial anisotropy axis. The band structure of paramagnetic $\mathrm{CoO}$ is similar to that of fcc $\mathrm{Co}$, except for some band narrowing due to the increased interatomic distance and some hybridization with oxygen states. ${ }^{2}$ However, when it became clear that the Co $3 d$ electrons are localized, ${ }^{3}$ emphasis shifted toward localized models. This refers, in particular, to the magnetic anisotropy of $\mathrm{CoO}$, which is usually considered as a crystal-field effect but has recently attracted renewed attention ${ }^{4}$ due to its nontrivial orbital-moment character. In this paper, we investigate how electron correlations affect the magnetic anisotropy, interpolating between the undercorrelated (band structure) and overcorrelated (localized) limit.

There are two extreme models of magnetic anisotropy. The point-charge model (Fig. 2) treats the system as an insulator and ascribes the anisotropy to crystal-field charges, ${ }^{5-8}$ whereas the itinerant limit involves delocalized orbitals formed from atomic orbitals. ${ }^{8,9}$ We are primarily interested in the involved orbitals, but it is worthwhile recalling that anisotropy also involves spin-orbit coupling. The calculations are usually performed using the Stevens coefficients and perturbation theory, but the orbitals in Fig. 2 are chosen to yield

${ }^{a)}$ Electronic mail: rskomski@neb.rr.com. zero and maximum orbital moment and anisotropy along the bond axis. This is because the elongated $\left|z^{2}\right\rangle$ and the flat $\left|x^{2}-y^{2}\right\rangle \pm i|x y\rangle$ shown in the figures have orbital moments of $m=0$ and $m=2$, respectively. ${ }^{8}$

In the $\mathrm{CoO}$ structure, each $\mathrm{Co}$ atom is coordinated by 6 $\mathrm{O}$ nearest neighbors and by 12 Co next-nearest neighbors. The hopping integrals between the Co $3 d$ and O $2 p$ orbitals are large, but the low energy of the oxygen orbitals means that there is little hybridization between $\mathrm{Co}$ and $\mathrm{O}$ orbitals. The resulting $3 d$ bands are fcc-like, and the bandwidth is largely determined by the hopping between next-nearest $\mathrm{Co}$ neighbors, with an oxygen hybridization contribution of somewhat more than $30 \%$. $^{2}$ Another difference between fcc

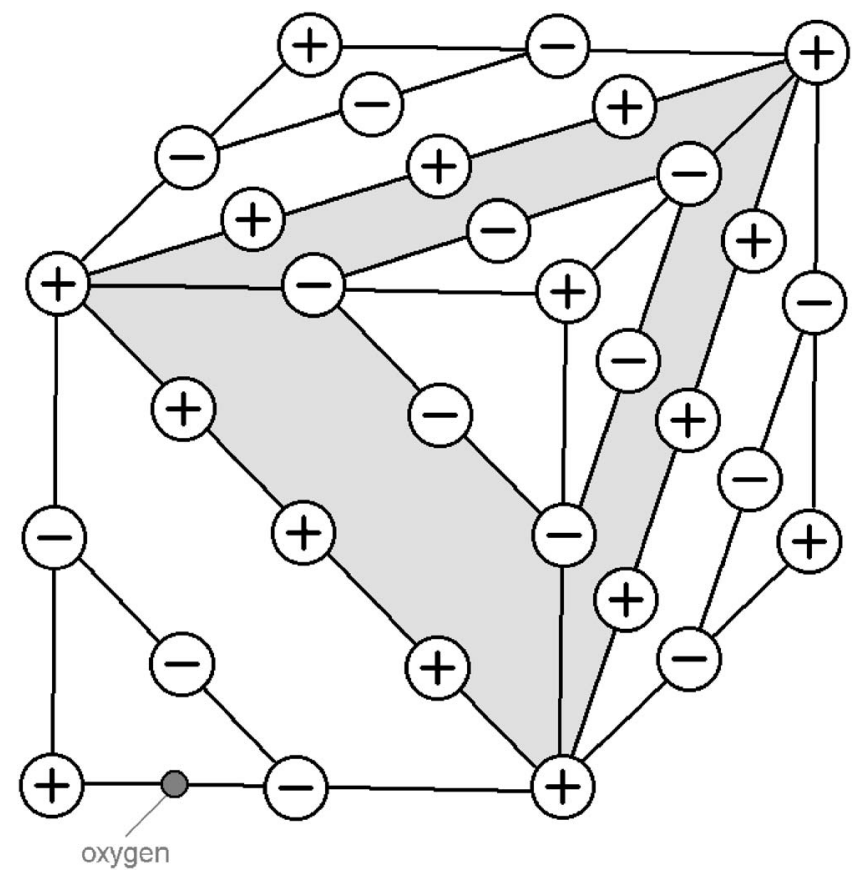

FIG. 1. Spin structure of antiferromagnetic CoO. The sign ( \pm ) indicates the spin direction $(\uparrow$ and $\downarrow)$ and the easy magnetization axis is along the cube edges. 

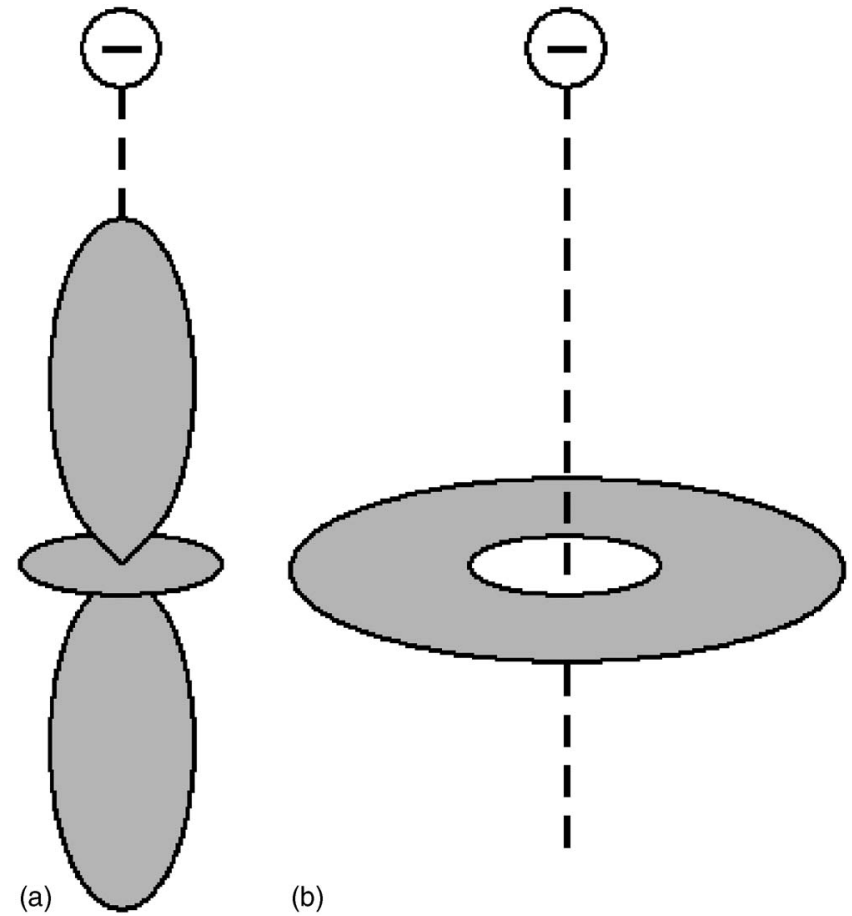

(b)

FIG. 2. Point-charge model of crystal-field interaction and magnetic anisotropy: (a) prolate orbital $(m=0)$ and (b) oblate orbital $(m=2)$. The case (b) is energetically more favorable, because neighboring atoms typically carry an negative charge (Ref. 10) and repulsively interact with the $d$ or $f$ electrons in the partially filled inner shells. This mechanism yields, for example, the huge magnetocrystalline anisotropy of rare-earth permanent magnets (Refs. 6 and 8)

$\mathrm{Co}$ and $\mathrm{CoO}$ is that the cubic crystal field changes sign, because the $\mathrm{O}$ atoms give rise to octahedral nearest-neighbor coordination. In other words, in $\mathrm{CoO}$ the energy of the $t_{2 g}$ states is lower than that of the eg states. This change is of secondary importance to the band structure but reverses the sign of the corresponding anisotropy contribution.

The minority electrons of antiferromagnetic $\mathrm{CoO}$ are largely confined to the Co (111) planes, in contrast to paramagnetic $\mathrm{CoO}$. The situation is reminiscent of the behavior of $\mathrm{Cu} 3 d^{9}$ in the $\mathrm{Cu}-\mathrm{O}$ planes of $\mathrm{La}_{2} \mathrm{CuO}_{4}$, but both the band structure and the effect of correlations are complicated by the presence of more than one electron or hole per site. Using a configuration-interaction (CI) approximation, we analyze the effect of $3 d^{8}-3 d^{6}$ bonds and their interplay with $3 d^{7}-3 d^{7}$ configurations. This mechanism yields a specific anisotropy contribution, different from but adding to the electrostatic or "point-charge" and LCAO-type ligand-field (or itinerant) anisotropy contributions. Figure 3 shows the corresponding tight-binding band structure of a Co (111), using the $d d \sigma$, $d d \pi$, and $d d \delta$ hopping integrals from Ref. 2. The small bandwidth, less than $2 \mathrm{eV}$, contains the reduced hybridization between $3 d$ orbitals located in adjacent (111) planes.

Correlation effects in solids are a considerable challenge, because they occur "on top" of the band structure. In a few cases, such as the $\mathrm{CuO}_{2}$ planes of $\mathrm{La}_{2} \mathrm{CuO}_{4}$, it is possible to restrict the consideration to a single band with one electron or hole per atom, corresponding to fluctuations between $\mathrm{Cu}$ $3 d^{9}$ and $3 d^{10}$ configurations. ${ }^{11}$ However, $\mathrm{CoO}$ has two Co $\downarrow$ electrons per atom in the minority band, corresponding to a

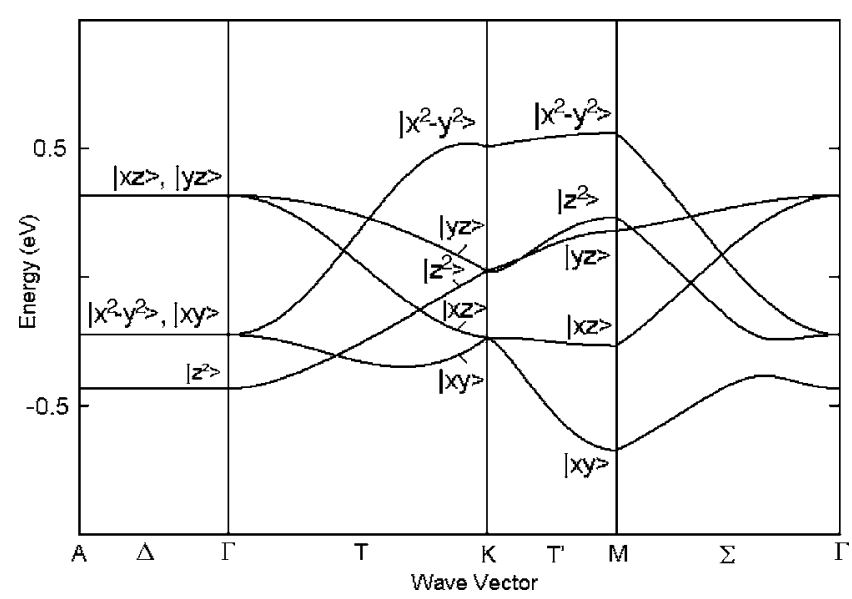

FIG. 3. Approximate band structure for Co minority spins in the (111) Co planes.

$3 d^{7}$ configuration. This means that $40 \%$ of the bands shown in Fig. 2 are filled, and we cannot restrict the consideration to low-lying states such as those formed from $|x y\rangle$ orbitals at the $M$ point.

Here, we take a somewhat different approach and focus on pairs of neighboring Co atoms. The replacement of eigenfunctions $|\psi\rangle$ by approximate eigenfunctions $|\phi\rangle$ can be considered as a case of downfolding. ${ }^{12-14}$ As emphasized by Löwdin, ${ }^{15}$ the Schrödinger equation $\mathbf{H}|\psi\rangle=E|\psi\rangle$ can be written as

$$
\mathbf{H}_{o}|\phi\rangle+\mathbf{V}|\chi\rangle=E|\phi\rangle
$$

and

$$
\mathbf{V}^{+}|\phi\rangle+\mathbf{H}_{r}|\chi\rangle=E|\chi\rangle .
$$

Here, $|\chi\rangle$ belongs to $|\psi\rangle$ but not to $|\phi\rangle$, and $\mathbf{H}_{r}$ and $\mathbf{V}$ are the respective diagonal and off-diagonal interactions involving $|\chi\rangle$. Substitution of $|\chi\rangle$ into Eq. (1a) and (1b) yields

$$
\mathbf{H}_{o}|\phi\rangle+\mathbf{V} \frac{1}{E-\mathbf{H}_{r}} \mathbf{V}^{+}|\phi\rangle=E|\phi\rangle .
$$

This equation is the type $\mathbf{H}_{\text {eff }}|\phi\rangle=E|\phi\rangle$, where $\mathbf{H}_{\text {eff }}$ describes the interaction of the bare states $|\phi\rangle$ with the environment $|\chi\rangle$.

The downfolding procedure leading to Eq. (2) is exact but leads to a complicated expression for $\mathbf{H}_{\text {eff }}$. A crude approach is to treat the matrix elements of $\mathbf{H}_{\text {eff }}$ as energydependent parameters. To investigate the effect of correlations on the crystal field, we choose the hybridized wave functions shown in Figs. 4(a) and 4(b). In Fig. 4(a), the interatomic hybridization between $z^{2}$ orbitals $(m=0)$ yields bonding and antibonding states $s$ and $s^{*}$, respectively, whereas in Fig. 4(b) the same occurs for $x y$ and $x^{2}-y^{2}$ orbitals $(m=2)$. Such hybridization exist in the (111) planes if we use an appropriately rotated coordinate frame. Figure 3 captures essential features of the problem, although anisotropy is obtained by summation over all pairs of orbitals, and we do not claim that the specific orbitals shown in Fig. 3 yield a disproportionally large anisotropy contribution.

In Fig. 3, the $\sigma$ and $\delta$ bonds yield to zero and easy-axis anisotropy, respectively. Note that $m=0$ ( $\sigma$ bond) implies 


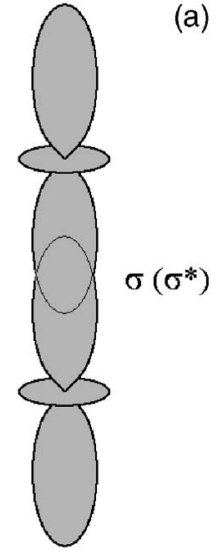

(b)

(c)
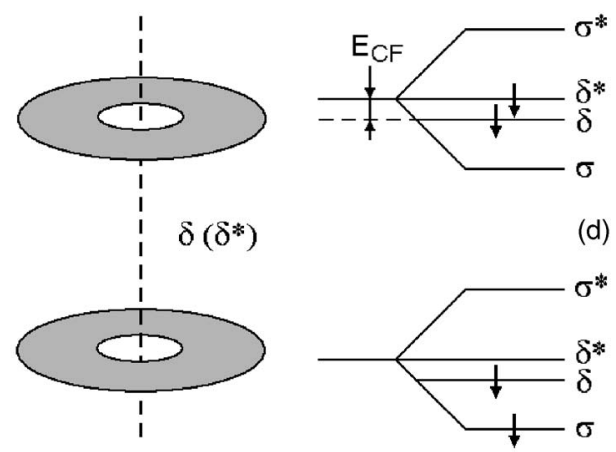

FIG. 4. Interatomic hybridization and crystal field: (a) $d d \sigma$ overlap, (b) $d d \delta$ overlap, and (c) and (d) two spin configurations derived from (a) and (b). Note that $d d \sigma$ hopping integrals are much larger than $d d \delta$ hopping integrals, and compared to Fig. 2, the case (a) has now the lower energy, because it amounts to bonding $\sigma$ orbital.

zero orbital moment, and therefore zero spin-orbit coupling and zero anisotropy. The Hund's rule interactions with fully occupied majority $(\uparrow)$ band ensure that all spin states are $\downarrow$, but there are several possibilities of accommodating the spins. This is a common feature of correlations, and a conceptually simple approach is actually the consideration of CI between different many-electron states. ${ }^{11}$ For strong correlations, the lowest lying configuration is that shown in Fig. 4(c). The occupancy of the $\sigma$ and $\sigma^{*}$ states is low lying, too, but is slightly less favorable due to the crystal-field splitting $E_{\mathrm{CF}}$.

Why does the second electron occupy the antibonding $\delta^{*}$ level rather than the bonding and low-lying $\sigma$ level, as in Fig. 4(d)? The reason is that Fig. 4(d) implies pronounced charge fluctuations, amounting to the coexistence of $3 d^{7}-3 d^{7}$ and $3 d^{6}-3 d^{8}$ configurations. This can be shown by explicit consideration of the wave functions $|\sigma\rangle=\left|\sigma_{T}\right\rangle+\left|\sigma_{B}\right\rangle,\left|\sigma^{*}\right\rangle=\left|\sigma_{T}\right\rangle$ $-\left|\sigma_{B}\right\rangle,|\delta\rangle=\left|\delta_{T}\right\rangle+\left|\delta_{B}\right\rangle,\left|\delta^{*}\right\rangle=\left|\delta_{T}\right\rangle-\left|\delta_{B}\right\rangle$, where the indices refer to the top and bottom orbitals shown in Fig. 4(a). The $\delta$ - $\delta^{*}$ configuration has the two-electron wave function $|\delta\rangle\left|\delta^{*}\right\rangle-\left|\delta^{*}\right\rangle|\delta\rangle=\left|\delta_{B}\right\rangle\left|\delta_{T}\right\rangle-\left|\delta_{B}\right\rangle\left|\delta_{T}\right\rangle$, that is, each of the two atomic $\delta$ orbitals (top and bottom) is single occupied. The same calculation for $|\sigma\rangle|\delta\rangle-|\delta\rangle|\sigma\rangle$ yields a $50 \%$ admixture of states such as $\left|\sigma_{T}\right\rangle\left|\delta_{T}\right\rangle-\left|\delta_{T}\right\rangle\left|\sigma_{T}\right\rangle$, meaning that both electrons are accommodated in the top or bottom atom. This costs correlation energy $(U)$, is energetically unfavorable, and causes the transition from Figs. 4(d) and 4(c).
From a many-electron point of view, each one-electron calculation corresponds to one Slater determinant where available low-lying one-electron states are filled like liquid which is poured into a jar, as in Fig. 4(d). A simple example is the tight-binding (or LCAO) approximation, where the one-electron states are linear combinations of atomic orbitals, but advanced local spin-density approximation (LSDA) electronic-structure calculations are based on the same principle. This includes LSDA $+U$ calculations, which contain a self-energy correction $(U)$ and yield improved predictions on a quantum-mechanical mean-field level. ${ }^{16}$ However, the treatment of specific correlation effects, such as spin-charge separation, ${ }^{17}$ requires the explicit or implicit consideration of several Slater determinants. ${ }^{11}$ In the present paper, this is done in an approximate way, by considering the two configurations Figs. 4(c) and 4(d), but a detailed analysis of configurations and their interactions in $\mathrm{CoO}$ remains a challenge to future research.

In summary, we have investigated how electron correlations affect the magnetocrystalline anisotropy of $\mathrm{CoO}$. Correlations suppress the charge fluctuations $\left(3 d^{6}\right.$ and $3 d^{8}$ configurations) associated with the formation of bonding states and thereby change the character of the crystal-field interactions responsible for magnetic anisotropy. The resulting scenario is intermediate between the electrostatic or pointcharge and itinerant (or LCAO-type ligand-field) mechanisms. In the highly simplified model of Fig. 3, this leads to a change from zero anisotropy to easy-axis anisotropy.

This research is supported by NSF MRSEC and NCMN.

${ }^{1}$ W. L. Roth, Phys. Rev. 110, 1333 (1958).

${ }^{2}$ L. F. Mattheiss, Phys. Rev. B 5, 290 (1972).

${ }^{3}$ L. F. Mattheiss, Phys. Rev. B 5, 306 (1972).

${ }^{4}$ R. J. Radwanski and Z. Ropka, Physica B 345, 107 (2003).

${ }^{5}$ F. Bloch and G. Gentile, Z. Phys. 70, 395 (1931).

${ }^{6}$ J. F. Herbst, Rev. Mod. Phys. 63, 819 (1991).

${ }^{7}$ R. Skomski, Philos. Mag. B 70, 175 (1994).

${ }^{8}$ R. Skomski and J. M. D. Coey, Permanent Magnetism (IOP, Bristol, 1999).

${ }^{9}$ H. Brooks, Phys. Rev. 58, 909 (1940).

${ }^{10}$ D. J. Newman and B. Ng, Rep. Prog. Phys. 52, 699 (1989).

${ }^{11} \mathrm{P}$. Fulde, Electron Correlations in Molecules and Solids (Springer, Berlin, 1991).

${ }^{12}$ W. R. L. Lambrecht and O. K. Andersen, Phys. Rev. B 34, 2439 (1986).

${ }^{13}$ O. K. Andersen and T. Saha-Dasgupta, Phys. Rev. B 62, R16219 (2000).

${ }^{14}$ R. W. Tank and C. Arcangeli, Phys. Status Solidi B 217, 89 (2000).

${ }^{15}$ P.-O. Löwdin, J. Chem. Phys. 19, 1396 (1951).

${ }^{16}$ O. Bengone, M. Alouani, P. Blöchl, and J. Hugel, Phys. Rev. B 62, 16392 (2000).

${ }^{17}$ B. I. Halperin, J. Appl. Phys. 101, 081601 (2007). 